\title{
Relationships of leisure-time physical activity and work ability between different occupational physical demands in adult working men
}

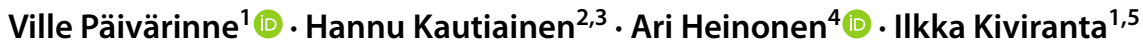

Received: 30 July 2018 / Accepted: 20 January 2019 / Published online: 31 January 2019

(C) The Author(s) 2019

\begin{abstract}
Purpose Leisure-time physical activity (LTPA) is known to be associated with positive health benefits, but the role of occupational physical demands remains inconsistent. The purpose of the current study was to assess the relationship between LTPA and work ability in different occupational physical activity (OPA) levels between young adult men.

Methods We performed physical activity measurements in work and leisure time with the long version of International Physical Activity Questionnaire (IPAQ) and work ability with the Work Ability Index (WAI) in 921 Finnish employed male volunteer participants. The participants were divided into LTPA tertiles I ( $<8 \mathrm{MET}$-h/week), II (8-28 MET-h/week), and III (> 28 MET-h/week) and OPA tertiles I (0 MET-h/week), II (<64 MET-h/week), and III ( $\geq 64 \mathrm{MET}$-h/week).

Results There was a significant relationship between LTPA and WAI in OPA tertiles (adjusted for age, alcohol consumption, working class status, BMI, and employment years). Moreover, each LTPA tertile showed significant linear associations with WAI $(P<0.001)$.

Conclusion LTPA is positively associated with work ability among young adult men. More specifically, the relationships between LTPA and WAI were significantly greater in physically demanding jobs than in more passive jobs. Our results indicate the importance of LTPA, particularly with individuals under higher work-related physical strain.
\end{abstract}

Keywords Exercise $\cdot$ MET $\cdot$ Questionnaires $\cdot$ Occupational physical demands · Occupational health

\section{Introduction}

The health benefits of physical activity (PA) are well documented, even though most of the results are restricted to leisure-time physical activity (LTPA) (Warburton et al. 2006; Lear et al. 2017). On the contrary, particularly in individuals with low fitness levels, growing evidence emphasizes the role of occupational physical activity (OPA) as not only

Ville Päivärinne

ville.paivarinne@helsinki.fi

1 Department of Orthopaedics and Traumatology, University of Helsinki, P.O. Box 63, Helsinki 00014, Finland

2 Primary Health Care Unit, Kuopio University Hospital, Kuopio, Finland

3 Folkhälsan Research Center, Helsinki, Finland

$4 \quad$ Faculty of Sport and Health Sciences, University of Jyväskylä, Jyväskylä, Finland

5 Helsinki University Hospital, Helsinki, Finland inadequate but detrimental to health, particularly among men (Li et al. 2013; Holtermann et al. 2016, 2018; Coenen et al. 2018). The vast majority of Finnish adults reportedly do not meet PA recommendations, which increases the risk for several diseases such as cardiovascular and musculoskeletal disorders (Biswas et al. 2015; Bennie et al. 2017). In addition, the imbalance of daily PA can decrease overall health and functionality with reduced work performance, while resulting in an increased risk for long-term sickness absence and premature retirement due to disability (Holtermann et al. 2012, 2016; Mänty et al. 2015; Søgaard and Sjøgaard 2017).

The concept of work ability relates to individual physical and mental capacities and the demands of the job, where it can be used as a tool by professionals for health promotion and disease and injury prevention (Tengland 2011). Previous evidence has shown that more physically active occupations, such as construction, have a higher risk of work-related disability than less physically demanding occupations (Alavinia et al. 2009). In Finland, a little less than a third of male 
employees consider their work as physically demanding (Kauppinen et al. 2013). In addition, work itself is insufficient to prevent decline in work ability; measures to promote work ability should start before middle age, especially among workers in physically demanding jobs (Ilmarinen et al. 1997). Work ability can be assessed by the Work Ability Index (WAI), which is a widely used and well-accepted self-reported instrument that measures health and functional capacity via a single dimension of work ability (Ilmarinen et al. 2005).

Even though the deleterious health effects of a sedentary lifestyle and the beneficial effects of PA in adult populations are currently widely acknowledged (Warburton et al. 2006; Biswas et al. 2015), further research between more specific dimensions, i.e. LTPA and WAI within domains of OPA, is warranted. Previous studies have presented a strong positive association between the lack of vigorous LTPA and poor WAI (van den Berg et al. 2009) and there has been relationship when achieving recommended LTPA levels and better WAI among sedentary workers (Nawrocka et al. 2017). In addition, previous study found an association between high LTPA levels and better work ability in middle-aged workers with physically demanding jobs (Calatayud et al. 2015). However, the evidence remains limited for an interaction between LTPA and OPA in work ability, particularly among young adult men.

The purpose of this cross-sectional, population-based study was to determine the association between LTPA and WAI in interaction with OPA dimensions in young adult men.

\section{Methods}

\section{Design and participants}

The participants for this cross-sectional, population-based study were collected in 2009 from five cohorts [birth years $1969(N=67), 1974(N=139), 1979(N=228), 1984$ $(N=229)$, or $1989(N=258)]$ of Finnish men, who had performed, withdrew from, or discontinued military service or had performed an alternative non-military service. Immigrants, imprisoned subjects, those with mental disorders, or the unemployed were excluded from the study. Initially, 1425 male participants who answered for the PA questionnaire were extracted as a population sample. Informed consent was obtained from all individual participants included in the study.

\section{Questionnaire}

A questionnaire was applied to record PA, work ability, health behavioral and functional capacity, musculoskeletal disorders, mental disorders, pain, and alcohol consumption. The questions were partly based on the Finnish Health 2000 study, the IPAQ questionnaire, and WAI questionnaire (Aromaa and Koskinen 2004; International Physical Activity Questionnaire team 2005; Rautio and Michelsen 2014).

\section{Physical activity}

The long version of the International Physical Activity Questionnaire (IPAQ-long) in the Finnish language was used to estimate the level of LTPA and OPA. The IPAQ comprises of four different detailed PA levels (work-related activity, leisure-time activity, transport-related activity, and domestic and garden activities) that require respondents to recall their PA over the past 7 days. In our study, we concentrated to work-related activity as OPA and leisure-time activity as LTPA, wherein both domains contained three levels of intensity (walking, moderate, or vigorous) to provide domain-specific scores. Both domains of activity are defined in metabolic equivalent minutes per week (MET-min/week) by multiplying the MET value for the activity ( 3.3 for walking, 4.0-6.0 for moderate-intensity activity, and 8.0 for vigorous-intensity activity) with duration (minutes) and frequency (days). After this, we converted values to more broadly used MET-hours per week (MET-h/week) (International Physical Activity Questionnaire team 2005). IPAQ is a valid and reliable instrument for assessing levels and patterns of PA (Hagstromer et al. 2006). Additionally, IPAQ has been culturally adapted for the Finnish population (Craig et al. 2003). More specific calculations and assessments of the IPAQ have been described previously (Päivärinne et al. 2018). Participants were divided into tertiles based on their total LTPA: I (<8 MET-h/week), II (8-28 MET-h/week), and III ( $>28 \mathrm{MET}-\mathrm{h} /$ week $)$ and total OPA: I (0 MET-h/ week), II ( $<64 \mathrm{MET}$-h/week), and III ( $\geq 64 \mathrm{MET}$-h/week).

\section{Work ability}

Participant work ability was assessed by the Work Ability Index (WAI), which is a self-reported instrument that assesses work ability and diagnoses, symptoms, and sickness absence to measure the health and functional capacity via a single dimension of work ability (Ilmarinen et al. 2005). The WAI consists of seven items regarding both physical and psychological aspects of work ability. Scores range from 7 (lowest) to 49 (highest). The points of the WAI form the basis for determining the level of work ability according to the following scales: 7-27 ("poor" work ability), 28-36 ("moderate" work ability), 37-43 ("good" work ability) and 44-49 ("excellent" work ability) (Ilmarinen et al. 2005; Rautio and Michelsen 2014). The WAI has been demonstrated 
to be a valid instrument for assessing work ability (Lundin et al. 2017).

\section{Other variables}

The participants' occupational status were asked using the question "Are you currently...?" with response options of employee, clerical employee, superior, expert, department manager, senior manager or other. Diagnosed disorders were inquired through several different illnesses and were only taken into account if they were diagnosed by a doctor (accidental injury, musculoskeletal disease, circulatory disease, respiratory disease, or mental health disorders) (Ilmarinen et al. 2005; Rautio and Michelsen 2014). Daily alcohol consumption and frequency (weekly/monthly) over the past 12 months were asked using the question "During the past 12 months, how often have you had a drink containing alcohol?" with response options of never, 6-7 times a week, 4-5 times a week, 2-3 times a week, once a week, once a month or less than once a month (Aromaa and Koskinen 2004). The Finnish guidelines for high-risk alcohol consumption levels for healthy adult males are considered as more than 6 drinks at once and 23-24 drinks per week (Kauhanen et al. 1992). The numeric rating scale (NRS), which is a reliable and valid instrument for assessing pain were used to assess general pain, neck pain, upper limb pain, low back pain and lower limb pain, was used to assess general pain, lower back pain, lower limb pain, neck pain, or upper limb pain (Hawker et al. 2011). Score was scaled through 0-10, where 0 referred to "no pain" and 10 to "pain as bad as it could be" (McCaffery and Beebe 1993). Participants' Body Mass Index (BMI) were calculated with a subject's weight in kilograms divided by the square of the subject's height in meters $\left(\mathrm{kg} / \mathrm{m}^{2}\right)$ (Khosla and Lowe 1967). Commonly used BMI ranges are underweight (below $18.5 \mathrm{~kg} / \mathrm{m}^{2}$ ), normal weight $\left(18.5-24.9 \mathrm{~kg} / \mathrm{m}^{2}\right)$, overweight $\left(25.0-29.9 \mathrm{~kg} / \mathrm{m}^{2}\right)$ and obese (over $30.0 \mathrm{~kg} / \mathrm{m}^{2}$ ) (World Health Organization Europe 2018).

\section{Statistical analyses}

Data are presented as means with standard deviations (SD) or as counts with percentages. The statistical significance for the linearity across three LTPA and OPA levels (tertiles) was tested using analysis of variance (ANOVA) with appropriate contrast (orthogonal polynomial) and Cochran-Armitage test (Altman 1991). A possible nonlinear relationship between WAI and the standardized or continuous LTPA was assessed using three-knot-restricted cubic spline regression models. The length of the distribution of knots was located by Harrell's default at the 10th, 50th, and 90th percentiles. Regression models included age, alcohol consumption, body mass index (BMI), employment years, and working class status as covariates. In case of violation of the assumptions (e.g., non-normality), a bootstrap-type method was used $(10,000$ replications) to estimate the standard error (Elfron and Tibshirani 1998); distribution of WAI index was skewed left. Effect sizes, eta-squared $\left(\eta^{2}\right)$, were calculated using the linear models (ANOVA). By convention, values of 0.01, 0.06, and 0.14 are deemed small, medium, and large effect sizes, respectively (Cohen 1988). The normality of variables was evaluated by the Shapiro-Wilk $W$ test. Stata 15.0 (StataCorp LP; College Station, Texas, USA) statistical package was used for the analysis.

\section{Results}

\section{Participant demographics}

The sample consisted of 921 male participants who were under part-time or full-time employment. Demographic and clinical characteristics of the study participants according to LTPA (MET-h/week) are shown in Table 1. A small but statistically significant linear relationship was observed between several variables. Between subject age and LTPA; older subjects had lower LTPA MET-h/week scores $(P=0.009)$. Furthermore, linear relationships across MET tertiles were observed between the subjects' BMI $(P=0.024)$, working class status $(P=0.040)$, employment years $(P=0.039)$, and presence of cardiovascular disorders $(P=0.001)$. Additionally, a linear relationship was observed between LTPA and weekly alcohol consumption $(P=0.005)$, general pain $(P=0.030)$, lower back pain $(P=0.036)$, neck pain $(P=0.001)$, upper limb pain $(P=0.001)$, and sick days during the last 12 months $(P=0.004)$.

\section{Activity scores}

In panel A of Fig. 1, age-, alcohol consumption-, BMI-, employment year-, and working class status-adjusted mean WAI are shown across the MET tertiles. After adjustment, LTPA associated with higher work ability $(P<0.001)$ $\left[\eta^{2}=0.03(95 \% 0.01-0.05)\right]$, whereas different OPA levels did not associate with WAI. No interaction was observed between LTPA and OPA (Fig. 1b) tertiles $\left[\eta^{2}=0.00(95 \%\right.$ $0.00-0.01)]$.

\section{Relationship between activity levels and work ability}

Figure 2 illustrates the cubic spline regression of change in WAI in relation to 1 SD per standardized LTPA MET-h/ week. In OPA tertile I, the WAI change per 1 SD was 0.93 (95\% confidence interval $[\mathrm{CI}] 0.32-1.54 ; P=0.003$ ). In tertile II, the WAI change per $1 \mathrm{SD}$ was $0.59(95 \%$ [CI] 
Table 1 Demographic and clinical characteristics of the participants $(N=921)$ divided into tertiles according leisuretime physical activity (MET-h/ week) levels
Fig. 1 a Work ability index (WAI) in leisure-time physical activity (LTPA) tertiles and occupational physical activity (OPA) tertiles adjusted for age, alcohol consumption, BMI, employment years, and working class status. b Adjusted mean of WAI across the LTPA in each OPA tertile. Means with $95 \%$ confidence intervals are shown. $P$ value shows linearity between the tertiles

\begin{tabular}{lcccc}
\hline & $\mathrm{I},(n=304)$ & $\mathrm{II},(n=311)$ & $\mathrm{III},(n=306)$ & $P$ value $^{\mathrm{a}}$ \\
\hline Age, mean (SD) & $33.2(6.3)$ & $32.7(5.9)$ & $31.6(6.4)$ & 0.009 \\
BMI, mean (SD) & $26.4(4.4)$ & $26.2(3.7)$ & $25.7(3.2)$ & 0.024 \\
Working class, $n$ (\%) & & & \\
Blue collar & $216(71.1)$ & $185(59.5)$ & $193(63.1)$ & 0.040 \\
Employment years, mean (SD) & $7.1(5.7)$ & $7.4(6.0)$ & $6.2(5.5)$ & 0.039 \\
Disorders, $n$ (\%) & & & & \\
Accidents & $29(10)$ & $34(11)$ & $36(12)$ & 0.38 \\
Musculoskeletal disorders & $60(20)$ & $67(22)$ & $56(18)$ & 0.66 \\
Cardiovascular disorders & $19(6)$ & $14(5)$ & $3(1)$ & 0.001 \\
Lung disorders & $11(4)$ & $14(5)$ & $14(5)$ & 0.56 \\
Mental disorders & $13(4)$ & $13(4)$ & $8(3)$ & 0.28 \\
Alcohol consumption per week, mean (SD) & $7.7(10.4)$ & $6.9(9.1)$ & $5.7(6.3)$ & 0.005 \\
Pain, NRS, mean (SD) & $1.80(2.05)$ & $1.59(1.96)$ & $1.44(1.86)$ & 0.030 \\
Low back pain & $1.80(2.19)$ & $1.61(2.04)$ & $1.43(2.01)$ & 0.036 \\
Lower limb pain & $0.90(1.62)$ & $0.92(1.74)$ & $0.87(1.48)$ & 0.82 \\
Neck pain & $1.88(2.19)$ & $1.65(2.11)$ & $1.30(1.76)$ & 0.001 \\
Upper limb pain & $1.07(1.97)$ & $0.63(1.46)$ & $0.59(1.38)$ & $<0.001$ \\
Sick days $>9$ (last 12 months), $n(\%)$ & $61(20)$ & $44(14)$ & $36(12)$ & 0.004 \\
Occupational physical activity, $n(\%)$ & & & & 0.016 \\
I (0 MET-h/week) & $86(28.3)$ & $110(35.4)$ & $116(37.9)$ & \\
II (<64 MET-h/week) & $99(32.6)$ & $107(34.4)$ & $90(29.4)$ & \\
III ( $\geq 64$ MET-h/week) & $119(39.1)$ & $94(30.2)$ & $100(32.7)$ & \\
\hline
\end{tabular}

$N R S$ numeric rating scale

${ }^{\text {a }} P$ for linearity across MET tertiles. Count variables calculated using Cochrane-Armitage test and mean values by Anova

${ }^{\mathrm{b}}$ Units per week

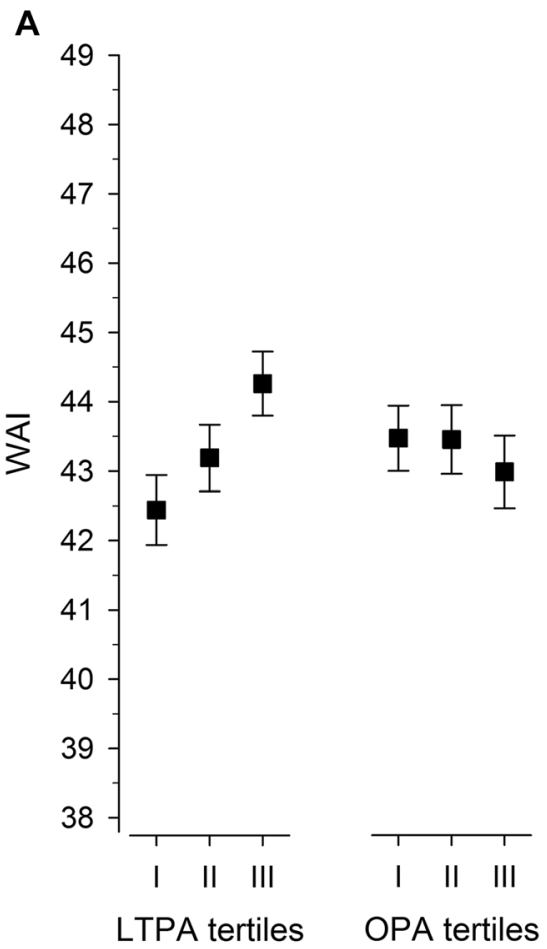




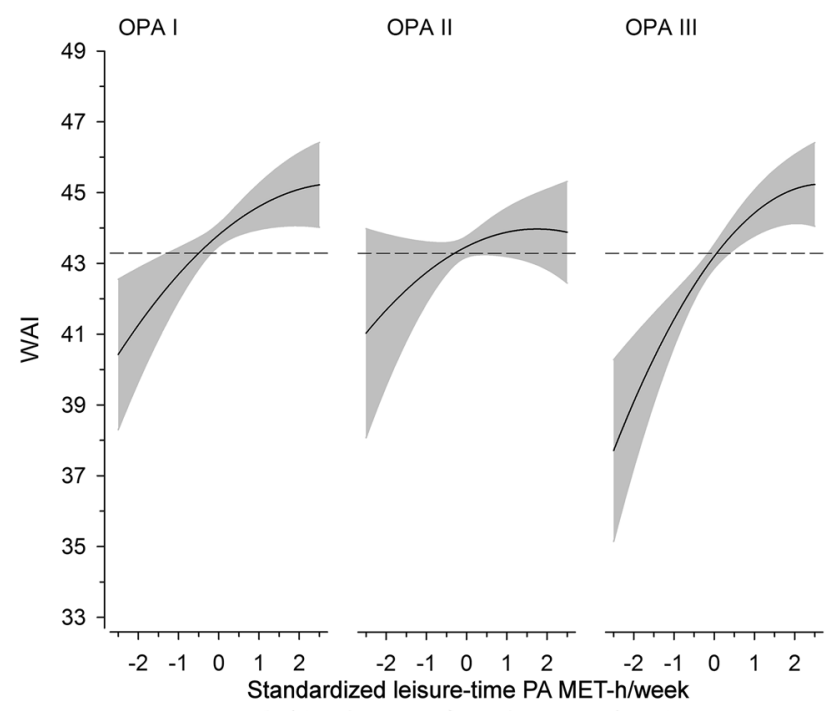

Fig. 2 Relationship between standardized leisure-time physical activity (MET-h/week) and work ability index (WAI) in different occupational physical activity (OPA) tertiles. The curves were derived from a three-knot restricted cubic splines regression model. The model was adjusted for age, alcohol consumption, working class status, BMI, and employment years. The gray areas represent 95\% confidence intervals. The dashed line indicates the mean value of all participants in WAI

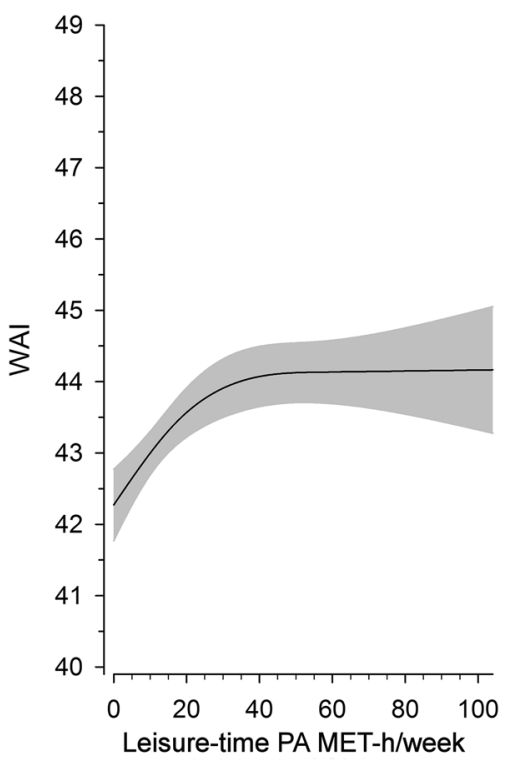

Fig. 3 Relationship between continuous leisure-time physical activity (MET-h/week) and work ability index (WAI). The curves were derived from a three-knot restricted cubic splines regression model. The model was adjusted for age, alcohol consumption, working class status, BMI, and employment years. The gray area represents $95 \%$ confidence intervals
- 0.14-1.32; $P=0.112)$. In tertile III, the WAI change per 1 SD was 1.51 (95\% CI 0.88-2.14; $P \leq 0.001)$ after adjusting for age, alcohol consumption, working class status, BMI, and employment years.

The cubic splines relationship between continuous LTPA and WAI is illustrated in Fig. 3. The regression curve showed a positive association between LTPA and WAI, particularly if weekly LTPA achieved approximately 40 MET-h/week.

\section{Discussion}

The results presented here indicate that among a population of healthy male subjects between 20 and 40 years, self-reported LTPA (MET-h/week) associated with better work ability, particularly in subjects with more physically demanding jobs. Furthermore, the results indicate that when exposed to different OPA levels, higher LTPA levels were positively associated with WAI. More specifically, LTPA had a significantly greater relationship between WAI in subjects with physically demanding jobs than in subjects with more sedentary jobs. This association was independent of age, BMI, alcohol consumption, working class status, and employment years.

\section{Previous findings}

Our findings are consistent with the systematic review of van den Berg et al. (2009), which describes a strong association between absence of vigorous LTPA and WAI in an adult population. Furthermore, LTPA has a positive relationship with WAI among a cohort of mostly female health care workers (Arvidson et al. 2013). There is also evidence from a randomized controlled study that physical exercise intervention improves work ability due to enhanced cardiorespiratory fitness (Kettunen et al. 2014). Moreover, evidence from a cross-sectional study revealed an association between high LTPA levels and better work ability in workers with physically demanding jobs (Calatayud et al. 2015). However, this study was conducted on middle-aged participants and a more heterogeneous population with different PA and work ability measurement methods. Information on combined associations of LTPA and work ability in different occupational physical demands is currently limited, particularly in young adult men.

\section{Interpretation of principal findings}

In our study, the greater relationship between high LTPA and excessive OPA in work ability could be partially explained by the maintaining and improving effects of LTPA on cardiorespiratory fitness and health, which is normally included with voluntary movement and a sufficient amount 
of recovery time (Warburton et al. 2006). In contrast, OPA has been considered to have longer periods and lower intensity levels of maximal aerobic capacity, while elevating the 24-h heart rate and blood pressure, which may even impair cardiovascular health (Korshøj et al. 2015; Holtermann et al. 2018). In the present study, high levels of LTPA could also play a part in improving the responsiveness and adaptation of cardiovascular health and heart rate recovery (Lamberts et al. 2010). Since excessive periods of high OPA are reported to prolong high heart rates, high OPA and low cardiorespiratory fitness could cause cardiovascular strain that may lead to cardiovascular disorders. Higher cardiovascular fitness could possibly prevent these outcomes (Korshøj et al. 2015; Holtermann et al. 2016) and thus, appear in individuals with less illnesses, sick days and overall better work ability. Additionally, LTPA has been shown to decrease the risk for long-term sickness absence (LTSA), whereas OPA has been shown to increase the risk for LTSA (Holtermann et al. 2012). Moreover, LTPA has previously associated with fewer disability pensions because of musculoskeletal disorders (Fimland et al. 2015). Our study showed that the number of sick days linearly decreased with increasing LTPA MET tertiles.

As higher LTPA is associated with better physical and musculoskeletal fitness (Warburton et al. 2006), higher LTPA could be beneficial for those who are exposed to physical workloads including heavy lifting with static and constrained postures and activities, possibly impairing physical health functioning (Holtermann et al. 2012; Mänty et al. 2015). In our study, the positive association of LTPA could appear in the form of better self-assessed work ability, particularly in subjects with physically demanding jobs. On the other hand, it could also refer to better pain self-efficacy (Denison et al. 2007) strategies or other health-enhancing effects in relation to intensity, duration, or type of contraction caused by LTPA (Søgaard and Sjøgaard 2017). These effects could help more physically active participants to cope and adapt with the physical demands of high OPA (Holtermann et al. 2018). In our study, this could explain the inverse linear relationship across MET tertiles and the incidence of general pain, lower back pain, neck pain, and upper limb pain. In addition, individuals with low self-efficacy due to pain-concentrated disorders are reported to have a high probability of avoiding PA in their daily routines (Rejeski et al. 1998). The terminology of musculoskeletal disorders (MSD) is considered as a broad term covering any nonspecific disorder characterized by pain or decreased functioning (Søgaard and Sjøgaard 2017). Therefore, because of the possibly lower muscular health level in the lower PA group, lower WAI scores and higher pain incidence could indicate functional impairments in the future.

In addition, our study presented a smaller but significant relationship between lower OPA levels and LTPA. Nawrocka et al. (2017) found a relationship between achieving recommended LTPA levels and WAI among white-collar workers. In the present study, even though the majority of participants were blue-collar workers, our results indicated a similar relationship in the group that had the lowest OPA level. Other studies have shown that sedentary workplace behavior combined with low LTPA is associated with work-related fatigue, musculoskeletal pain, and a higher risk for heart failure or burnout (de Vries et al. 2017; Naczenski et al. 2017). In the present study, higher LTPA and musculoskeletal fitness could particularly reduce work-related pain in the lowest OPA group, which is caused by time spent in unnatural and static or standing positions (Coenen et al. 2016). Such pain reduction could relate to better WAI.

In the present study, the participants were men of 20-40 years who had good work ability and were expected to have many employment years in the course of their career. However, the risk of subsequent retirement due to disability and mortality associated with lack of PA is expected to increase in middle age (Lear et al. 2017). Our current study revealed an inverse linear relationship between LTPA and working years, meaning that subjects that had more years under employment were also less physically active. In addition, the positive relationship between work ability and LTPA, particularly at the highest OPA, could be considered noteworthy as we have previously observed that participants who recorded the greatest share of their PA from work (61\%) had, in contrast, the lowest level of LTPA (16\%) (Päivärinne et al. 2018). Our present study adds value to our previously reported importance of LTPA, particularly in occupations that are physically demanding. As reported previously, individual factors (such as aging and lack of PA) may be associated with midlife transition and an increased risk of retirement due to disability (Lahti et al. 2016).

\section{Relevance of findings}

Our results provide important public health implications. Almost 30\% of male employees in Finland in 2013 considered their work as physically demanding; this rate is presumably even higher in other countries (Kauppinen et al. 2013). Additionally, the present findings provide valuable information on the associations between LTPA and work ability at different OPA levels, particularly in young adult men. Despite the strong evidence for the benefits of PA, there is need for studies that assess the multidimensional behaviors of PA, such as LTPA and OPA and their association with work ability, which could help create strategies for preventing sickness absence or premature retirement due to disability while improving the health-enhancing effects of PA for workers. 


\section{Strengths and limitations}

Our study has several strengths. We used validated and widely employed questionnaires, such as the IPAQ long form to calculate LTPA and occupational PA along with the WAI. Our data were collected and analyzed from a random, homogeneous sample of young adult men, which allowed us to make reliable generalizations. We also recruited a relatively large sample of young Finnish adult men. However, there are limitations that should be taken into consideration. Due to the cross-sectional design of the study, the exposure and outcome are simultaneously assessed and, therefore, it is not possible to establish a true cause-and-effect relationship. Second, the subjective method of self-assessing LTPA and WAI may have resulted in reporting bias that could affect the outcome. Third, caution is advised when generalizing these results to women.

\section{Conclusion}

In conclusion, LTPA has a positive association with work ability in young adult men. More specifically, the relationship between LTPA and WAI was significantly greater in physically demanding jobs than in more passive jobs. Even for the crosssectional nature of the study, due to fairly large and homogeneous sample size and commonly used questionnaires, our results present a valid implication for the positive effects of LTPA in workers under higher work-related physical strain. The present findings provide valuable information on the associations between LTPA and work ability at different OPA levels, particularly in young adult men. More studies are warranted to support the findings that could help create strategies for preventing sickness absence or premature retirement while improving the health-enhancing effects of PA for workers.

Acknowledgements Open access funding provided by University of Helsinki including Helsinki University Central Hospital. This study was funded by the Social Insurance Institute of Finland (ref: 16/26/2009), the Scientific Advisory Board for Defense (ref: 630/70.03.01/2015, 474/70.03.00/2016, 50/70.03.01/2018), the Centre for Military Medicine, and the Finnish Cultural Foundation (Kymenlaakso Regional Fund; Olavi and Alli Pietikäinen).

\section{Compliance with ethical standards}

Conflict of interest The authors have no conflicts of interest to disclose.

Ethical approval The study protocol (Dnro 267/13/03/00/09) was approved by the Coordinating Ethics Committee of the Helsinki University Hospital. Written informed consent was obtained from all participants prior to enrollment. The content is solely the responsibility of the authors and does not necessarily represent the official views of the funders. All authors approved the manuscript and this submission.

Open Access This article is distributed under the terms of the Creative Commons Attribution 4.0 International License (http://creativeco mmons.org/licenses/by/4.0/), which permits unrestricted use, distribution, and reproduction in any medium, provided you give appropriate credit to the original author(s) and the source, provide a link to the Creative Commons license, and indicate if changes were made.

\section{References}

Alavinia SM, de Boer AG, van Duivenbooden JC, Fringssen MH, Burdorf A (2009) Determinants of work ability and its predictive value for disability. Occup Med 59(1):32-37

Altman D (1991) Practical statistics for medical research. Champman and Hall, London

Aromaa A, Koskinen S (2004) Health and functional capacity in Finland: baseline results of the health 2000 health examination survey. Publications of the National Public Health Institute. http:// www.ktl.fi/health2000/index.uk.html. Accessed 7 Dec 2017

Arvidson E, Borjesson M, Ahlborg G, Lindegard A, Jonsdottir IH (2013) The level of leisure time physical activity is associated with work ability - a cross sectional and prospective study of health care workers. BMC Public Health 13:855. https://doi. org/10.1186/1471-2458-13-855

Bennie JA, Pedisic Z, Suni JH, Tokola K, Husu P, Biddle SJH, Vasankari T (2017) Self-reported health-enhancing physical activity recommendation adherence among 64,380 Finnish adults. Scand J Med Sci Sports 27(12):1842-1853

Biswas A, Oh PI, Faulkner GE, Bajaj RR, Silver MA, Mitchell MS, Alter DA (2015) Sedentary time and its association with risk for disease incidence, mortality, and hospitalization in adults: a systematic review and meta-analysis. Ann Intern Med 162(2):123-132

Calatayud J, Jakobsen MD, Sundstrup E, Casana J, Andersen LL (2015) Dose-response association between leisure time physical activity and work ability: cross-sectional study among 3000 workers. Scand J Public Health 43(8):819-824

Coenen P, Willenberg L, Parry S, Shi JW, Romero L, Blackwood DM, Maher CG, Healy GN, Straker LM (2016) Associations of occupational standing with musculoskeletal symptoms: a systematic review with meta-analysis. Br J Sports Med 52(3):176-183

Coenen P, Huysmans M, Holtermann A, Krause N, van Mechelen W, Straker L, van der Beek A (2018) Do highly physically active workers die early? A systematic review with meta-analysis of data from 193696 participants. Br J Sports Med 52:1320-1326

Cohen J (1988) Statistical power analysis for the behavioral sciences, 2nd edn. Lawrence Erlbaum, Hillsdale

Craig CL, Marshall AL, Sjostrom M, Bauman AE, Booth ML, Ainsworth BE, Pratt M, Ekelund U, Yngve A, Sallis JF, Oja P (2003) International physical activity questionnaire: 12-country reliability and validity. Med Sci Sports Exerc 35(8):1381-1395

de Vries JD, van Hooff ML, Guerts SA, Kompier MA (2017) Exercise to reduce work-related fatigue among employees: a randomized controlled trial. Scand J Work Environ Health 43(4):337-349

Denison E, Åsenlöf P, Sandborgh M, Lindberg P (2007) Musculoskeletal pain in primary health care: subgroups based on pain intensity, disability, self-efficacy, and fear-avoidance variables. J Pain 8(1):67-74

Elfron B, Tibshirani R (1998) An introduction to the bootstrap. Chapman and Hall/CRC, New York

Fimland M, Vie G, Johnsen R, Nilsen T, Krokstad S, Bjørngaard J (2015) Leisure-time physical activity and disability pension: 9 years follow-up of the HUNT Study, Norway. Scand J Med Sci Sports 25:e558-e565. https://doi.org/10.1111/sms.12369 
Hagstromer M, Oja P, Sjostrom M (2006) The international physical activity questionnaire (IPAQ): a study of concurrent and construct validity. Public Health Nutr 9(6):755-762

Hawker GA, Mian S, Kendzerska T, French M (2011) Measures of adult pain: Visual analog scale for pain (VAS pain), numeric rating scale for pain (NRS pain), McGill pain questionnaire (MPQ), short-form McGill pain questionnaire (SF-MPQ), chronic pain grade scale (CPGS), short form-36 bodily pain scale (SF-36 BPS), and measure of intermittent and constant osteoarthritis pain (ICOAP). Arthritis Care Res 63(11):240-252

Holtermann A, Hansen JV, Burr H, Sogaard K, Sjogaard G (2012) The health paradox of occupational and leisure-time physical activity. Br J Sports Med 46(4):291-295

Holtermann A, Marott JL, Gyntelberg F, Søgaard K, Mortensen OS, Prescott E, Schnohr P (2016) Self-reported occupational physical activity and cardiorespiratory fitness: Importance for cardiovascular disease and all-cause mortality. Scand J Work Environ Health 42(4):291-298

Holtermann A, Krause N, van der Beek AJ, Straker L (2018) The physical activity paradox: six reasons why occupational physical activity (OPA) does not confer the cardiovascular health benefits that leisure time physical activity does. Br J Sports Med 52:149-150

Ilmarinen J, Tuomi K, Klockars M (1997) Changes in the work ability of active employees over an 11-year period. Scand J Work Environ Health 23(1):49-57

Ilmarinen J, Tuomi K, Seitsamo J (2005) New dimensions of work ability. Int Congr Ser 1280:3-7

International Physical Activity Questionnaire team (2005) Guidelines for data processing and analysis of the international physical activity questionnaire (IPAQ). http://www.ipaq.ki.de/scoring.pdf. Accessed 4 Dec 2018

Kauhanen J, Julkunen J, Salonen JT (1992) Coping with inner feelings and stress: heavy alcohol use in the context of alexithymia. Behav Med 18(3):121-126

Kauppinen T, Mattila-Holappa P, Perkiö-Mäkelä M, Saalo A, Toikkanen J, Tuomivaara S, Uuksulainen S, Viluksela M, Virtanen S (2013) Occupation and health in finland 2012. Helsinki, Finland: Finnish Institute of Occupational Health. http://www.julkari.fi/ handle/10024/134951. Accessed 28 Feb 2018

Kettunen O, Vuorimaa T, Vasankari T (2014) 12-mo intervention of physical exercise improved work ability, especially in subjects with low baseline work ability. Int J Environ Res Public Health 11(4):3859-3869

Khosla T, Lowe C (1967) Indices of obesity derived from body weight and height. Brit J Prev Soc Med 21:122-128

Korshøj M, Lidegaard M, Kittel F, Van Herck K, De Backer G, De Bacquer D, Holtermann A, Clays E (2015) The relation of ambulatory heart rate with all-cause mortality among middle-aged men: a prospective cohort study. PLoS One 10(3):e0121729. https://doi. org/10.1371/journal.pone.0121729

Lahti J, Holstila A, Mänty M, Lahelma E, Rahkonen O (2016) Changes in leisure time physical activity and subsequent disability retirement: a register-linked cohort study. Int J Behav Nutr Phys Act 13(1):99. https://doi.org/10.1186/s12966-016-0426-2

Lamberts RP, Swart J, Capostagno B, Noakes TD, Lambert MI (2010) Heart rate recovery as a guide to monitor fatigue and predict changes in performance parameters. Scand J Med Sci Sports 20(3):449-457
Lear SA, Hu W, Rangarajan S, Gasevic D, Leong D, Iqbal R, Casanova A, Swaminathan S, Anjana RM, Kumar R, Rosengren A, Wei L, Yang W, Chuangshi W, Huaxing L, Nair S, diaz R, Swidon H, Gupta R, Mohammadifard N, Lopez.Jaramillo P, Oguz A, Zatonska K, Seron P, Avezum A, Poirier P, Teo K, Yusuf S (2017) The effect of physical activity on mortality and cardiovascular disease in 130000 people from 17 high-income, middle-income, and lowincome countries: the PURE study. Lancet 390(10113):2643-2654

Li J, Loerbroks A, Angerer P (2013) Physical activity and risk of cardiovascular disease: what does the new epidemiological evidence show? Curr Opin Cardiol 28(5):575-583

Lundin A, Leijon O, Vaez M, Hallgren M, Torgen M (2017) Predictive validity of the work ability index and its individual items in the general population. Scand J Pub Health 45(4):350-356

Mänty M, Kouvonen A, Lallukka T, Lahti J, Lahelma E, Rahkonen O (2015) Changes in working conditions and physical health functioning among midlife and ageing employees. Scand J Work Environ Health 41(6):511-518

McCaffery M, Beebe A (1993) Pain: clinical manual for nursing practice. V.V. Mosby Company, Baltimore

Naczenski L, de Vries J, van Hooff M, Kompier M (2017) Systematic review of the association between physical activity and burnout. J Occup Health 59(6):477-494

Nawrocka A, Garbaciak W, Cholewa J, Mynarski W (2017) The relationship between meeting of recommendations on physical activity for health and perceived work ability among white-collar workers. Eur J Sport Sci 18(3):415-422

Päivärinne V, Kautiainen H, Heinonen A, Kiviranta I (2018) Relations between subdomains of physical activity, sedentary lifestyle, and quality of life in young adult men. Scand J Med Sci Sports 28(4):1389-1396

Rautio M, Michelsen T (2014) WAI-how to use the Work ability index questionnaire. Finnish Institute of Occupational Health 2nd revised edition. Tammerprint Oy, Tampere

Rejeski WJ, Ettinger WH Jr, Martin K, Morgan T (1998) Treating disability in knee osteoarthritis with exercise therapy: a central role for self-efficacy and pain. Arthritis Care Res 11(2):94-101

Søgaard K, Sjøgaard G (2017) Physical activity as cause and cure of muscular pain: Evidence of underlying mechanisms. Exerc Sport Sci Rev 45(3):136-145

Tengland P (2011) The concept of work ability. J Occup Rehabil 21(2):275-285

van den Berg TI, Elders LA, de Zwart BC, Burdorf A (2009) The effects of work-related and individual factors on the work ability index: a systematic review. Occup Environ Med 66(4):211-220

Warburton DE, Nicol CW, Bredin SS (2006) Health benefits of physical activity: the evidence. CMAJ 174(6):801-809

World Health Organization Europe (2018) Body mass index-BMI. http://www.euro.who.int/en/health-topics/disease-prevention/ nutrition/a-healthy-lifestyle/body-mass-index-bmi. Accessed 7 Dec 2018

Publisher's Note Springer Nature remains neutral with regard to jurisdictional claims in published maps and institutional affiliations. 